\title{
Evolution of Cementite in Hypoeutectoid Steel Wire during Cold Work Hardening in Rolling Process
}

\author{
Wang Bojian $^{1}$, Ge Xiaochen ${ }^{2}$, Xiao Qiang ${ }^{1}$, Liu Shifeng ${ }^{1}$ \\ ${ }^{1}$ School of Metallurgy Engineering, Xi'an University of Architecture and Technology, Xi'an, China \\ ${ }^{2}$ Engineering Practice and Training Center, Northwestern Polytechnic University, Xi'an, China
}

\section{Email address:}

jkdwangbj@sina.com (Wang Bojian)

\section{To cite this article:}

Wang Bojian, Ge Xiaochen, Xiao Qiang, Liu Shifeng. Evolution of Cementite in Hypoeutectoid Steel Wire during Cold Work Hardening in Rolling Process. International Journal of Mineral Processing and Extractive Metallurgy. Vol. 3, No. 3, 2018, pp. 60-64.

doi: 10.11648/j.ijmpem.20180303.12

Received: May 28, 2018; Accepted: July 5, 2018; Published: October 27, 2018

\begin{abstract}
Using $\Phi 1.80 \mathrm{~mm}$ steel wire drawn from $\Phi 6.5 \mathrm{~mm}$ steel wire was drawn to $\Phi 1.80 \mathrm{~mm}$ as raw material. Using cold three-rolling process, we got flat wire which size was $0.75 \mathrm{~mm}$ x $3.17 \mathrm{~mm}$ by cold three-pass rolling process. Researches shown that 60 steel wire has good plastic deformation ability during cold work processing. The tensile strength and micro-hardness shows linear growth characteristics in the early first stage of cold rolling, then they have exponential growth characteristics in later rollings. That is because in the first rolling process the cementite eventually dissolved in large quantities as the ferrite is turned, deformed, refined and broken. The solution mechanism of cementite is the Gibbs-Thomson effect, i. e. interface energy mechanism.
\end{abstract}

Keywords: Rolling, Hypoeutectoidsteel, Cementite, Dissolution

\section{Introduction}

In the 1960s, the "white corrosion layer" appeared on the rail surface. It is proven to be caused by the constant impact of the wheel on the rail surface to dissolves the carburization of the steel rail surface [1]. Belous et al. proved that the carburizing body can be partially dissolved during deformation of drawing [2]. Hono et al. found observed that the complete dissolve of carburizing experience was completely dissolved in the tensile strain of 5.1 [3]. Minna's research proves that the solubility of cementite also increase the dependent variable. A series of research results show that the deformation and dissolution of cementite can occur during the deformation process of pearlite steel, but the mechanism of dissolution is currently controversial [4]. There are two views interpretations on the dissolution mechanism of cementite: (1) There is a large attraction between carbon atoms and dislocation. In the process of deformation, the dislocation slip will tow away the captured carbon atoms and transport them to the ferrite film layer, known as the carbon drag effect [5]; (2) The plastic deformation causes the cementite to form small flakes, which leads to the increase of the interfacial freedom free energy of cementite and matrix. This leads to an increase in the amount of carbon dissolved, which dissolves the carburizing body, known as the Gibbs-Thomson effect [6].

At present, in the current industrial production, using the cold drawing steel wire which was eutectoid steel wire or hypereutectic steel wire to prepare the flat wire by drawing eutectoid steel wire or hypereutectic steel wire through cold rolling process to $\Phi 1.80 \mathrm{~mm}$. These flat wires are mainly used to make cleaning brush. The tensile strength of flat wire is greater than $1350 \mathrm{MPa}$. In this paper, we studied the evolvement rule of cementite in hypoeutectoid steel wire, and the evolvement rule of cementite has the effect of evolvement rule on tensile strength and micro-hardness of cementite.

\section{Experimental Materials and Methods}

The chemical composition of $\Phi 60$ wire $6.5 \mathrm{~mm}$ is shown in table 1 . We prepared $\Phi 1.80 \mathrm{~mm}$ steel wire by pickling, phosphating, saponification using water tank drawing machine to achieve $\Phi 1.80 \mathrm{~mm}$ steel wire. The total reduction rate during the process is $92.33 \%$. The $\Phi 60$ steel flat wire was prepared by using the two-roll three-rolling mechanism 
method, as shown in table 2.

Table 1. The chemical composition of 60 steel wire (wt \%).

\begin{tabular}{lllllllll}
\hline Element & $\mathbf{C}$ & $\mathbf{S i}$ & $\mathbf{M n}$ & $\mathbf{P}$ & $\mathbf{S}$ & $\mathbf{C r}$ & $\mathbf{N i}$ & $\mathbf{C u}$ \\
\hline Content & 0.60 & 0.22 & 0.30 & 0.016 & 0.015 & 0.075 & 0.015 & 0.02 \\
\hline
\end{tabular}

Table 2. The rolling process of flat wire.

\begin{tabular}{llll}
\hline Pass & Thickness & Reduction per draft & Total reduction \\
\hline 1 & 1.22 & $32.22 \%$ & $32.22 \%$ \\
2 & 0.80 & $34.43 \%$ & $55.56 \%$ \\
3 & 0.75 & $6.25 \%$ & $58.33 \%$ \\
\hline
\end{tabular}

According to national standard GB/T228.1-2010, we carried out room temperature tensile test using wdw-100 10-ton electronic drawing machine with $160 \mathrm{~mm}$ sample length, $50 \mathrm{~mm}$ standard distance, and $1 \mathrm{~mm} / \mathrm{min}$ stretching speed. The Vickers hardness test was conducted by Wolbert 401MVD digital display microscopic according to GB/ t4340.1-1999. The jsm-6390 scanning electron microscope is used for tissue analysis and secondary electron imaging. The metallographic sample etch was a concentration of $4 \%$ nitric acid alcohol.

\section{Experimental Results}

The changes of tensile strength and Vickers hardness of flat wire are shown in figure 1 and figure 2 respectively. It can be seen from the figure that the tensile strength and Vickers hardness have the same changing rule as deformation degree increases. During the first rolling, the increase of tensile strength and Vickers hardness show linear growth. After the second and third times rolling, the increase of tensile strength and Vickers hardness accelerated, showing the trend of exponential growth.

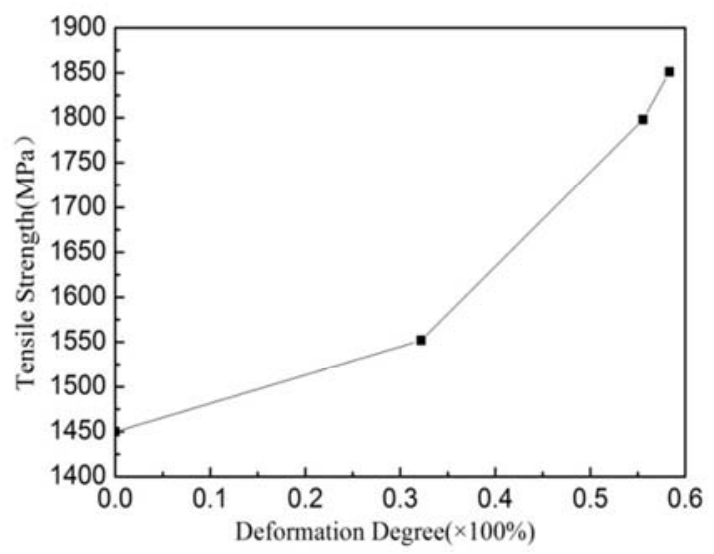

Figure 1. Change of tensile strength of flat wire.

The organization texture of steel wire before rolling is shown in figure 3: The cementite of A region presents an obvious "S" shape. The thickness of cementite is not even, and the direction angle between cementite and drawing direction is larger than that of steel wire drawing. Some of the cementite has been broken into small quantities of granules of varying sizes but very few. In B area of the cementite, and the drawing direction angle is less than $45^{\circ}$ Angle. The cementite is thin and relatively uniform, with some of the flakes ruptured during drawing. The cementite in $\mathrm{C}$ region is almost parallel to the drawing direction. The thickness of cementite is the thinnest in this case, and the area is the largest, while with some pearlite is broken into small short films.

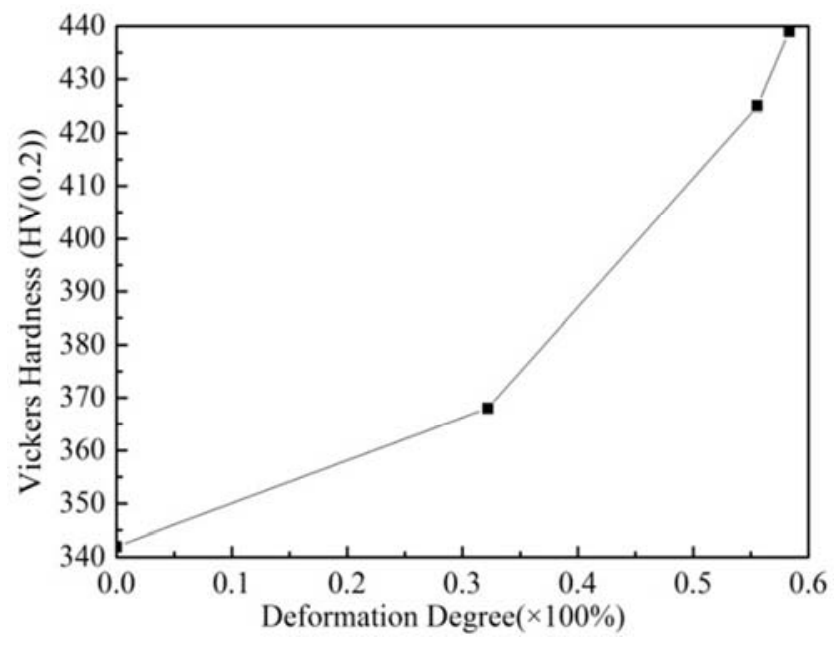

Figure 2. Change of Vickers hardness of flat wire.

After the first rolling, texture $\mathrm{A}, \mathrm{B}$ and $\mathrm{C}$ in the original organization still exist. The area proportion of area A decreases, and the cementite in A region becomes thinner and more curved. Part of the cementite ruptures at the maximum bending degree. The Angle between the pearlite and the rolling direction decreases, and in some case are even parallel to the rolling direction. The shorter cementite forms a short rod or granule after the slip deformation and fracture. Some pearlite particles are dissolved into smaller particles in deformation. Some of the pearlite can continue to deform and stretch along the rolling direction, resulting in a thin sheet. The pearlite in area $\mathrm{B}$ becomes thinner after rolling deformation. The pearlite and the rolling direction form a smaller Angle, and the pearlite tends to be more parallel to the rolling direction. The pearlite continues to fracture at the same time. The area proportion of $\mathrm{C}$ is increasing, and the original laminar layer is broken and partially dissolved, as shown in figure 4.

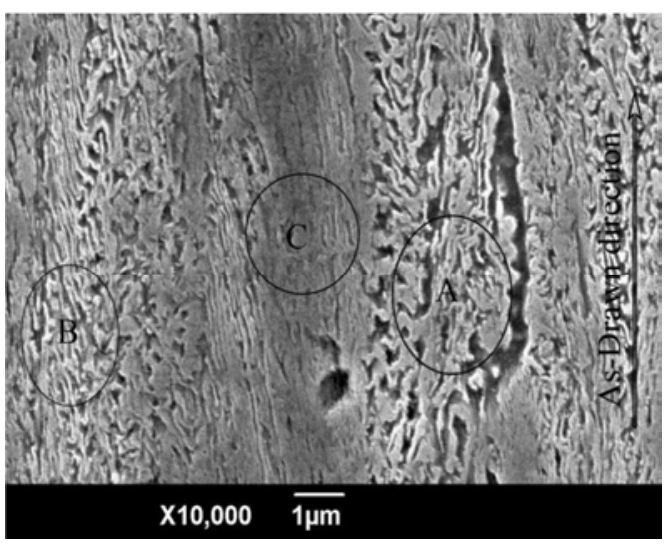

Figure 3. SEM microstructure of flat wire. 


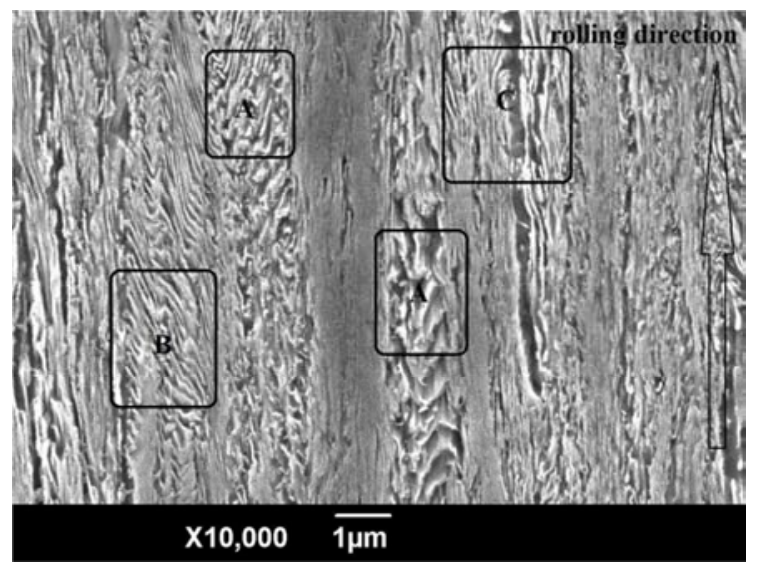

Figure 4. SEM microstructure after the first pass rolling.

After the second rolling, most of cementite is tending to parallel to rolling direction. Area A and area B are not strictly distinguishable. A small amount of bending pearlite can be observed in region $\mathrm{A}$. The broken cementite in area $\mathrm{B}$ continues to be refined and broken. The area proportion of $\mathrm{C}$ is increased rapidly increasing, and a large number of pearlite is further thinned and fragmented, as shown in figure 5.

After the third rolling, the cementite difference of area A and area $\mathrm{B}$ tends to disappear completely. The cementite is extremely refined. Thickness is about $40 \mathrm{~nm}$, and the partially refined cementite is dissolved as shown in figure 6.

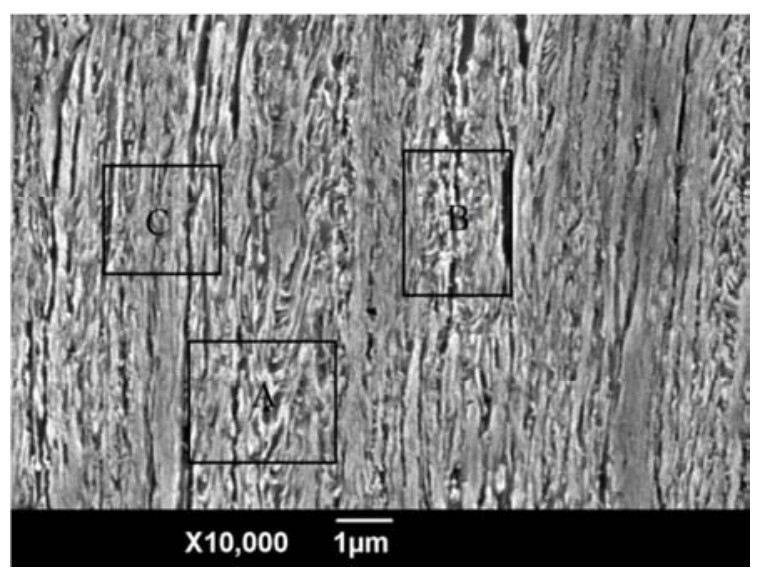

Figure 5. SEM microstructure after the second pass rolling.

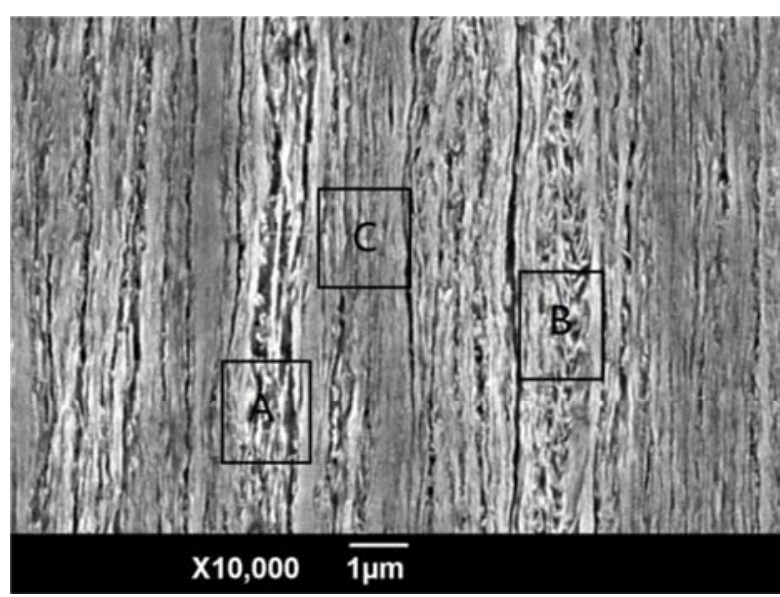

Figure 6. SEM microstructure after the third rolling.
This phenomenon can be seen in Figure. 7, after cold rolling the microstructure of the steel wire is almost all thin plate ferrite and the ferrite is parallel to the rolling direction. It is very difficult to observe the cementite in the microstructure after rolling. There is no cementite in the ferrite interface. Figure 8 shows the cementite in the steel wire. After cold rolling, cementite content is very low. Cementite is almost wrapped by ferrite body. The cementite is distributed in a long granular dispersion in the substrate parallel to the rolling direction. The boundary of the cementite is surrounded by a large number of dislocation locations. The study indicates that the cementite is deformed under the condition of large deformation. The cementite is changed by bending, turning and crushing. Figure 9 shows a cementite after rolling. This is because in the process of rolling deformation, there is a certain Angle of cementite in the original drawing microstructure. In order to meet the deformation of ferrite, the cementite is deformed. When the cementite deformation reaches a certain degree, the most severe bends will fracture. Figure 10 shows the broken cementite. In the process of deformation, the cementite is refined with the increasing of rolling deformation. In some regions, the cementite shows shear fracture, and finally the cementite is broken and dissolved.

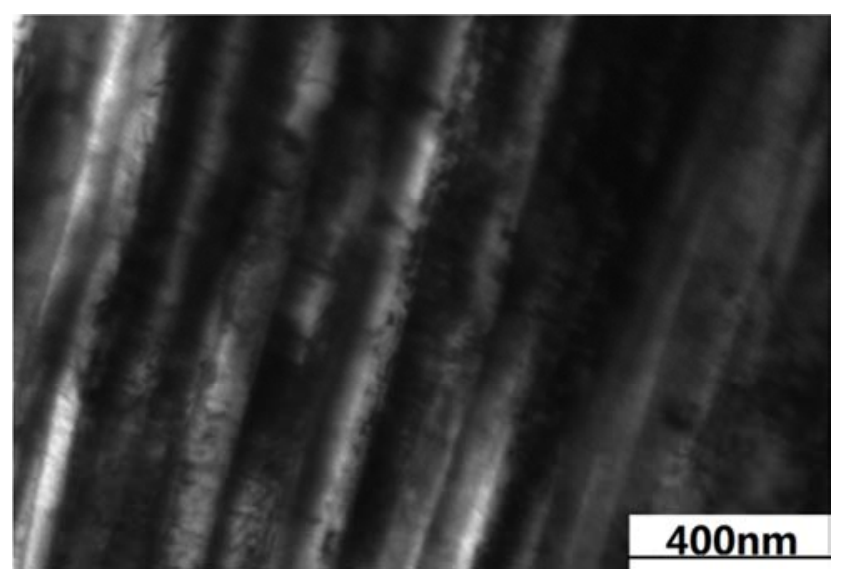

Figure 7. The TEM microstructures of steel wire after three-pass rolling process.

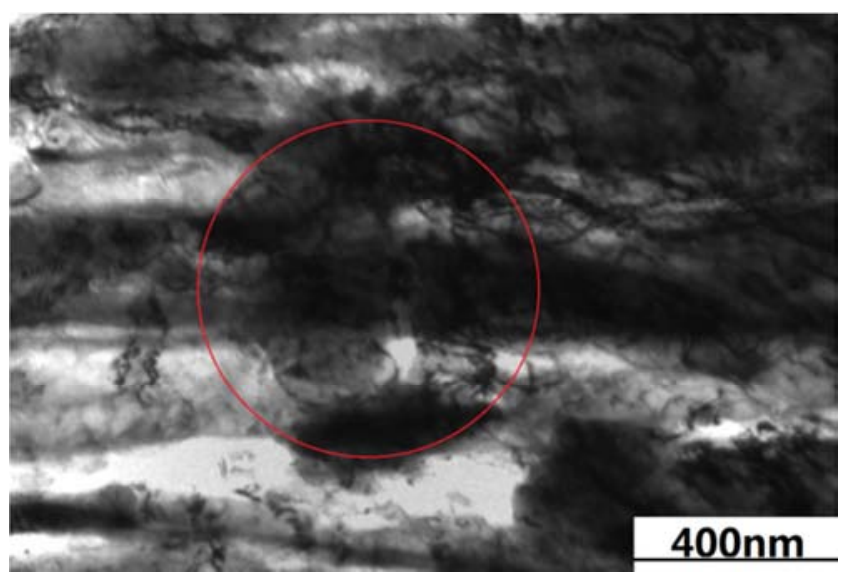

Figure 8. The TEM microstructures of steel wire after three-pass rolling process (cementite). 


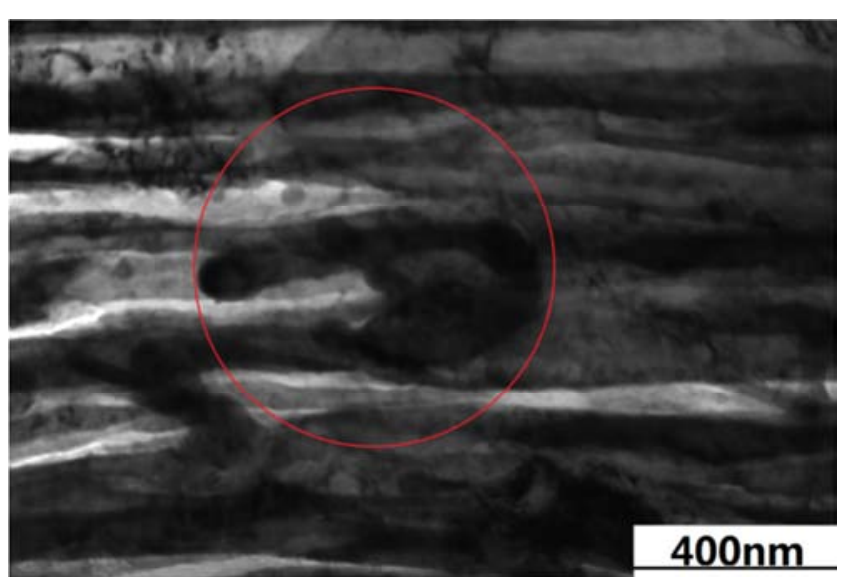

Figure 9. The TEM microstructures of steel wire after three-pass rolling process (deformation cementite).

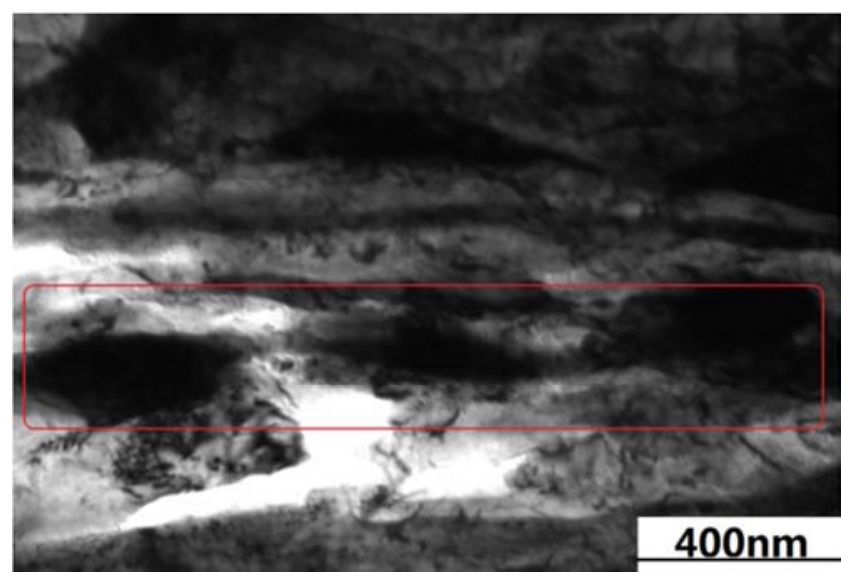

Figure 10. The TEM microstructures of steel wire after three-pass rolling process (fractured cementite).

\section{Analysis and Discussion}

Embury and Fisher put forward the direct relationship formula between the degree of deformation and intensity: $\sigma=\sigma_{0}+K \exp (\varepsilon / 4)$. That is to say, as the degree of deformation increases, the intensity increases [7]. Multiple experiments have proved that the calculated results are in good agreement with the experimental results for minor strain. This has been proved by many experiments. Using the data shown in this paper to calculate the above formula, we found that the data is basically aligned with above formula after the first rolling. However, after the second and third degree of deformation, the calculated data was is lower than the actual value. The phenomenon indicates that the reinforcement mechanism has changed, which could be related to the dissolution of carburizing body in the later stage of deformation.

When the cumulative rolling deformation is low, the strengthening mode of flat steel wire is mainly fine grain strengthening and dislocation strengthening. The mechanism of these two reinforcement methods is the dislocation slipping and the plugging product, and the dislocation density will increases dramatically in this stage. If the carburizing solution is due to the dragging and dropping of carbon atoms, a large number of cementite should be dissolved in this stage, then resulting in a sharp increase in tensile strength. However, the tensile strength was is found to be relatively slow at this stage. This indicates that the slip of dislocation is not the cause of mass dissolution of cementite. When the cumulative deformation of rolling is increased to a certain extent, the tensile strength shows an exponent increasing trend, while the smaller carburized part disappears. This indicates that the cementite of this stage has resulted in a significant increase in mechanical properties due to dissolution.

After analyzing the evolution law of microstructure and the change of tensile strength, the interface energy mechanism can be used to explain the process of carburizing during the rolling process. It mainly consists of the following two points: (1) in the initial stage of deformation, the cementite mainly happens to be small and broken refined and broken, resulting in a large number of fine particles or small pieces;(2) the rapid increase of tensile strength occurred occurs after the cementite was is broken up. The cementite that is extremely fragmented and tiny no longer fulfills critical size requirements, so they cannot exist independently. The size of carburized particles or small pieces is less than the critical grain size of the cementite. This type of cementite has a very high surface energy. According to the Thomson-Gibbs effect, the cementite will dissolve due to its inability to be independent and stable.

\section{Conclusion}

In the experiment, the strength of steel wire by coldworking shown two stage change with increase the degree of deformation. In the process of rolling, the cementite shows obvious rotation, parinization refining and dissolution. This makes the tensile strength and Vickers hardness of the steel wire grow exponentially in the latter stage. The dissolution mechanism of cementite is the interface energy mechanism, namely the Gibbs-Thomson effect.

\section{References}

[1] Xiao Qiang, Wang Bojian, Cao Meng. Research on situation of Forcible-dissolution and Precipitation Phenomenon at Room Temperature Under Severe Plastic Deformation [J]. Hot Working Technology, 2012, 41(20):41-44.

[2] M. V. Belous, V. T. Cherepin, Phys. Met. Metall. 1961, 12, 685-695.

[3] K. Hono, M. Ohnuma, M. Murayama, Cementite decomposition in heavily drawn pearlite steel wire, Scripta Mater., 2001, 977, 44-49.

[4] Min Na. Investigation on Phase Transformation during Severe Plastic Deformation and Aging in Eutectoid Steels [D]. Shanghai: Shanghai Jiao Tong University, 2007.

[5] A. V. Korznikov, Y. V. Ivanisenko, D. V. Laptionok, et al. Influence of severe plastic deformation on structure and phase composition of carbon steel [J]. Nanostructured Materials, 1994, 4(2): 159-167. 
[6] J. Languillaume, G. Kapelski, B. Baudelet. Cementite dissolution in heavily cold drawn pearlitic steel wires [J]. ActaMaterialia, 1997, 45(3):1201-1212.

[7] Embury J D, Fisher RM. The structure and properties of drawn pearlite [J]. ActaMetal, 1966, 14:147-153.

[8] Liu Ga, Wang Bojian, Bai He. Study on low temperature spheroidizing annealing process for severe plastic deformation steel wires [J]. Hot Working Technology, 2007, 36(22), 73-76.
[9] Ge XiaoChen, Wang BoJlian, et al. Hardness analysis on oil hardened and tempered flat wire for brush $[\mathrm{J}]$ Heat Treatment of Metals, 2015, 40(1), 201-203.

[10] Xiao Qiang, Wang BoJian, Liu ShiIFeng, et al. Microstructure and properties of annealed steel wire prepared by severe plastic deformation [J]. Heat Treatment of Metals, 2013, 38(6):6-9. 